BNL-114060-2017-JA

File \# 95041

\title{
Artifact mitigation of ptychography integrated with on-the-fly scanning probe microscopy
}

\author{
X. Huang, H. Yan, M. Ge, H. Ozturk, E. Nazaretski, I. K. Robinson, and Y. S. Chu
}

Submitted to: Applied Physics Letters

July 11, 2017

Photon Sciences Department

Brookhaven National Laboratory

\author{
U.S. Department of Energy \\ USDOE Office of Science (SC), \\ Basic Energy Sciences (BES) (SC-22)
}

Notice: This manuscript has been authored by employees of Brookhaven Science Associates, LLC under Contract No. DE- SC0012704 with the U.S. Department of Energy. The publisher by accepting the manuscript for publication acknowledges that the United States Government retains a non-exclusive, paid-up, irrevocable, world-wide license to publish or reproduce the published form of this manuscript, or allow others to do so, for United States Government purposes. 


\section{DISCLAIMER}

This report was prepared as an account of work sponsored by an agency of the United States Government. Neither the United States Government nor any agency thereof, nor any of their employees, nor any of their contractors, subcontractors, or their employees, makes any warranty, express or implied, or assumes any legal liability or responsibility for the accuracy, completeness, or any third party's use or the results of such use of any information, apparatus, product, or process disclosed, or represents that its use would not infringe privately owned rights. Reference herein to any specific commercial product, process, or service by trade name, trademark, manufacturer, or otherwise, does not necessarily constitute or imply its endorsement, recommendation, or favoring by the United States Government or any agency thereof or its contractors or subcontractors. The views and opinions of authors expressed herein do not necessarily state or reflect those of the United States Government or any agency thereof. 


\title{
Artifact mitigation of ptychography integrated with on-the-fly scanning probe microscopy
}

\author{
Xiaojing Huang, ${ }^{1}$ Hanfei Yan, ${ }^{1}$ Mingyuan Ge, ${ }^{1}$ Hande Öztürk, ${ }^{1}$ \\ Evgeny Nazaretski, ${ }^{1}$ Ian K. Robinson, ${ }^{2,3,4}$ and Yong S. Chu ${ }^{1}$ \\ ${ }^{1}$ National Synchrotron Light Source II, Brookhaven National Laboratory, Upton, NY 11973, USA* \\ ${ }^{2}$ Condensed Matter Physics and Materials Department, \\ Brookhaven National Laboratory, Upton NY 11973, USA \\ ${ }^{3}$ Research Complex at Harwell, Didcot, Oxfordshire OX11 ODE, UK \\ ${ }^{4}$ London Centre for Nanotechnology, University College London, London, WC1H 0AH, UK
}

(Dated: June 19, 2017)

\begin{abstract}
We report our experiences with conducting ptychography simultaneously with X-ray fluorescence measurement using the on-the-fly mode for efficient multi-modality imaging. We demonstrate that the periodic artifact inherent to the raster scan pattern can be mitigated using a sufficiently fine scan step size to provide an overlap ratio $>70 \%$. This allows us to obtain transmitted phase contrast images with enhanced spatial resolution from ptychography while maintaining the fluorescence imaging with continuous-motion scans on pixelated grids. This capability will greatly improve the competence and throughput of scanning probe X-ray microscopy.
\end{abstract}

Scanning probe X-ray microscopy is a powerful imaging method that simultaneously evokes multiple contrast mechanisms, including absorption, phase, fluorescence, diffraction and spectroscopy. These signals carry versatile information, and enable a suite of analytical tools to reveal a comprehensive view of the specimen under study. Correlative imaging using multiple contrast mechanisms can significantly leverage the information obtained in the images. With the steady progress on fabricating highresolution X-ray optics [1-5] and developing advanced microscopes [6-8], the achievable spatial resolution and detection sensitivity is continuously improving. Ptychography [9], as a scanning version of coherent diffraction imaging method, shares almost the same data acquisition scheme as a typical scanning probe microscope measurement, such as scanning X-ray transmission microscope (STXM) [10]. By scanning the specimen across a confined illumination probe with overlapped scanning spots and collecting far-field diffraction patterns using a pixelated area detector allows the complex-valued transmission function of the specimen to be reconstructed with a spatial resolution beyond the probe size [11-13]. The structural information provided by the absorption and phase contrast ptychography images complements other imaging channels that are recorded simultaneously, such as element maps from fluorescence [14], chemical states from spectroscopy [15] or crystalline orientations from diffraction. The recovered illumination function from ptychography reconstruction can also be used to improve the resolution of fluorescence images using probe deconvolution methods $[16,17]$. Scanning probe microscopy allows the integration of ptychography with other imaging channels within one single scan, providing the so-called multi-modality visualization capability [18].

The synergy between ptychography and scanning probe microscopy motivates further developments on how to efficiently combine these two techniques. The recent adaption of the on-the-fly scan scheme to ptychography has significantly improved the data acquisition efficiency by replacing step scans with continuous-motion scans [19-22]. This on-the-fly scan mode invokes the potential "periodicity pathology" problem arising from the symmetric scan trajectory, as a scanning probe measurement typically follows a raster scan pattern, which naturally gives meshed pixels to form two-dimensional images. In ptychography, it is reported that the periodicity of the raster scan pattern can introduce serious periodic artifacts in the obtained image [23]. This periodic artifact can be removed by choosing scan patterns without periodicity [24-26], but the customized scan patterns require changing the movement step sizes or movement directions between adjacent scan points. These scan patterns can not be easily accommodated in an on-the-fly scheme, and the scanning probe microscopy measurements with these patterns don't directly give pixelated images on a Cartesian coordinate without pixel interpolation for the other imaging channels. A scan trajectory with gradually varying row offsets was proposed for fly scan ptychography measurement [21], but it dose not directly provide pixelated images with other contrast mechanisms neither. The overlap ratio has been reported to play a critical role in governing the obtained image quality in ptychography [27-29], however its impact on the periodic artifact has not been discussed yet. In this study, we explore the possibility to mitigate the periodic artifact in ptychography reconstruction while acquiring data following regular mesh patterns as in typical scanning probe microscopy measurements. We demonstrate that the artifact can be significantly reduced if sufficient overlap redundancy is provided with fine scanning steps.

Through numerical simulations, we first evaluated the existence of periodic artifacts in ptychography operated in an on-the-fly scheme with mesh scan patterns. Diffraction patterns were initially generated with steps finer 

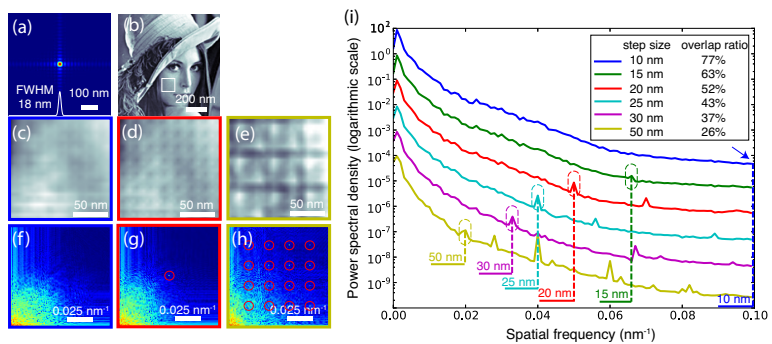

FIG. 1. Simulation results illustrating the impact of the scan step size on the periodic artifact in ptychography reconstructed images operated in the on-the-fly scheme with Poisson noise. (a) The illumination probe used in the simulation has side fringes in both vertical and horizontal directions, with a FWHM of $18 \mathrm{~nm}$. (b) The reconstructed phase image using the simulated data with $10 \mathrm{~nm}$ scan step size. (c)(d)(e) The zoom-in views of the reconstructed phase images over the region indicated by the white box in (b) with $10 \mathrm{~nm}, 20 \mathrm{~nm}$ and $50 \mathrm{~nm}$ scan step sizes, respectively. The evidence of the periodic artifacts is gradually mitigated with finer scan step size. (f) (g)(h) The Fourier modulus of the reconstructed phase images with $10 \mathrm{~nm}, 20 \mathrm{~nm}$ and $50 \mathrm{~nm}$ scan step sizes. Only one quadrant is displayed in logarithmic scale. The characteristic peaks associated to scan periodicities are highlighted by red circles. (i) The power spectral density calculated from reconstructed images with various scan step size. The plots are displaced by one order of magnitude relative to each other for clear illustration. The blue arrow points to the expected peak position for $10 \mathrm{~nm}$ periodicity.

than the target scan step size, and then the adjacent data frames were summed to simulate the blurry effect caused by continuous motion [19, 30]. A Poison noise was added to the data frames. The illumination probe was generated by wave propagation from a square pinhole. The peak had a full-width-at-half-maximum (FWHM) size of $18 \times 18 \mathrm{~nm}$, and the probe carries weak fringes in both horizontal and vertical directions to mimic side lobes in a realistic focused beam, as shown in Fig. 1 (a). Six diffraction datasets were generated using this probe with scan step sizes varying from $10 \mathrm{~nm}$ to 50 $\mathrm{nm}$. Each data frame had $128 \times 128$ pixels with $55 \mu \mathrm{m}$ pixel size. The illumination wavelength and detectorto-sample distance were set to give $5 \mathrm{~nm}$ reconstruction pixel size. 5 illumination modes were used in the reconstruction process. Each dataset was reconstructed from random guesses of both the probe and object functions using 500 iterations of Difference Map algorithm [11]. A typical reconstruction converged within 20 iterations. The outputs of the last 100 iterations were averaged to give the final recovered images. Fig. 1 (b) shows the reconstructed phase image using the simulated data with $10 \mathrm{~nm}$ scan step size. A zoom-in view of the obtained phase images shows that the periodic artifact is clearly seen with $50 \mathrm{~nm}$ scanning step (Fig. 1 (e)), and it is gradually mitigated with finer scanning steps (Fig. 1 $(c)(d))$. The periodic artifacts give characteristic peaks at the corresponding spatial frequencies in the Fourier modulus of the reconstructed phase images, as shown in Fig. 1 (g) and (h). Fig. 1 (i) plots the power spectral density curves of the Fourier modulus of reconstructed phase images with six simulated steps. The intensity of the characteristic peaks tends to decrease with the scan step size of $15 \mathrm{~nm}$, which is slightly below the FWHM size of the probe. For ptychography reconstruction, a more meaningful parameter representing the sampling condition is the 2D overlap ratio [31], which can be quantified as $o=\sum\left[\left|p\left(\vec{r}-\overrightarrow{r_{i}}\right) \| p\left(\vec{r}-r_{i+1}\right)\right|\right] / \sum\left[|p(\vec{r})|^{2}\right]$, where $p\left(\vec{r}-\overrightarrow{r_{i}}\right)$ and $p\left(\vec{r}-r_{i+1}\right)$ are the probes at two adjacent scan points and the summation is over all the pixels of the probe image. In the simulation setup, a $15 \mathrm{~nm}$ scan step size corresponds to an overlap ratio of $\sim 63 \%$, where the periodic artifact start to be noticeably mitigated. We note that this overlap ratio emphasizes the overlay of probe pixels with high intensities and it does not describe the actual number of overlapped nonzero probe pixels, which explains why the reconstruction does not fail completely when the calculated overlap ratio is below $50 \%$.

To validate these simulation results, we conducted a series of on-the-fly measurements at the Hard X-ray Nanoprobe (HXN) beamline of the National Synchrotron Light Source II (NSLS-II). The incident $12 \mathrm{keV}$ X-rays were focused by a pair of multilayer Laue lenses (MLL) to sub-20 nm. The coherent illumination was selected by a second source aperture placed about $15 \mathrm{~m}$ in front of the nano-focusing optics. The optics and microscope system to generate such a focus and perform scanning probe measurement are described elsewhere $[4,8,32,33]$. The sample used in the experiment contained gold nanoparticles, prepared by depositing a $20 \mathrm{~nm}$ thick layer of gold film onto a silicon substrate and then annealing the film at $800^{\circ} \mathrm{C}$ for 8 hours. The gold film became dewetted to isolated sub-micron crystals with various width and thickness. We selected one gold crystal of $\sim 500 \mathrm{~nm}$ wide and $\sim 200 \mathrm{~nm}$ thick, and collected four $2 \mathrm{D}$ scans in onthe-fly mode using $100 \times 100,75 \times 75,50 \times 50$, and $25 \times 25$ mesh patterns covering the same $1 \times 1 \mu \mathrm{m}$ area, which give $10 \mathrm{~nm}, 13.3 \mathrm{~nm}, 20 \mathrm{~nm}$ and $40 \mathrm{~nm}$ step sizes, respectively. A three-element silicon drift detector (Vortex-ME3) was placed perpendicularly to the incident beam to collect the gold fluorescence signals, and the far-field diffraction data was acquired by a pixelated area detector (Merlin) with $55 \mu \mathrm{m}$ pixel size at a distance of $0.5 \mathrm{~m}$ downstream the sample. The dwell time and deadtime for each scan point are 0.1 second and 0.005 second, respectively. The 0.1 second exposure time was determined to use the full dynamic range of the Merlin detector. A $186 \times 186$ pixel array was cropped from each diffraction frame and fed into 500 iterations of difference map algorithm to recover complex-valued real-space images, which results in a 5 $\mathrm{nm}$ pixel size.

The diffraction data collected in the on-the-fly mode is 
blurred due to the continuous sample motion. It has been previously demonstrated that this effect can be corrected by including multiple probe modes into the reconstruction process to represent an equivalent incoherent illumination condition assuming a static sample scanned by a moving beam [19-22]. We therefore used five illumination modes in reconstructing the ptychography datasets, and the relative power of the fifth mode was found to drop down to $\sim 1 \%$ for all scan step sizes. A reconstruction with a single illumination mode was performed first to provide an initial guess of the primary mode. The other four modes were initiated by randomly translating the primary probe within the range defined by the scan step size. The reconstructed modes were then orthogonalized using the single value decomposition method. The dropping of the primary mode power and the rising of higher-order powers both indicate that the effective coherence condition is degraded with larger fly-scan step sizes.
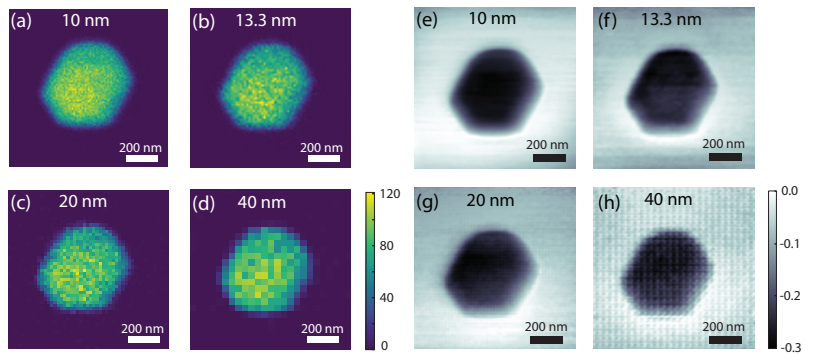

FIG. 2. Simultaneous X-ray fluorescence and ptychography imaging of a gold nanoparticle measured in on-the-fly mode. (a)-(d) The fitted gold $\mathrm{L} \alpha_{1}$ fluorescence maps with various scan step sizes over the same area. The scale bar is fluorescence counts per 0.1 second. (e)-(h) The phase images reconstructed by ptychography with multiple illumination modes to correct the diffraction data blur caused by fly-scan. The periodic artifact starts to be visible with $20 \mathrm{~nm}$ scanning step, and is readily apparent with $40 \mathrm{~nm}$ step size.

Fig. 2 shows the fluorescence images (a)-(d) and ptychographically reconstructed phase images (e)-(h) of the gold particle with various scan step sizes. The six-fold facets are clearly visible in the obtained images using both methods. The image pixel size of the fluorescence pictures is determined by the scan step size, thus the image quality is deteriorated with larger steps. While the ptychography reconstruction pixel size is maintained at 5 $\mathrm{nm}$ for all datasets, the image quality does not degrade as significantly as in the fluorescence case. However, as the on-the-fly scan followed regular mesh patterns, the periodic artifact starts to become visible with $20 \mathrm{~nm}$ scan step size, and is readily apparent with $40 \mathrm{~nm}$ step size.

The existence of periodic artifacts are further evaluated in Fourier space. Fig. 3 shows the Fourier modulus of the ptychography reconstructed phase images. The characteristic peaks associated with the periodicity of the scanning pattern clearly show up with $20 \mathrm{~nm}$ and $40 \mathrm{~nm}$
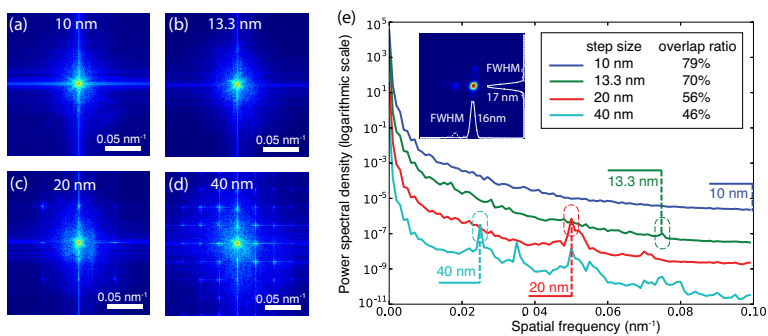

FIG. 3. Periodic artifact in reconstructed images. (a)-(d) The Fourier modulus of ptychographically reconstructed realspace images as shown in Fig. 2. Characteristic peaks associated to the periodicity of the scan pattern are clearly shown with $20 \mathrm{~nm}$ and $40 \mathrm{~nm}$ scan step size. (e) The power spectral density of reconstructed images demonstrate that the characteristic peak from periodic artifacts fades away until the scan step is less than $13.3 \mathrm{~nm}$ and the overlap ratio is above $70 \%$. The plots are displaced by one order of magnitude relative to each other for clear illustration. The primary probe mode reconstructed from the dataset with $10 \mathrm{~nm}$ scan step size is shown in the inset, which has a FWHM dimension of $16 \times 17$ nm.

scanning steps. In the power spectral density plots (Fig. 3 (e)), we can identify a peak corresponding to $13.3 \mathrm{~nm}$ periodicity for the reconstruction for the dataset with 13.3 $\mathrm{nm}$ scan step size, although this artifact can be merely recognized in either the recovered real-space image (Fig. 2 (f)) or its Fourier modulus (Fig. 3 (b)). It is also noticeable that the peak associated with $13.3 \mathrm{~nm}$ periodicity is at least one order-of-magnitude weaker than those in reconstructions from the datasets with $20 \mathrm{~nm}$ and $40 \mathrm{~nm}$ steps. This suggests that the periodic artifact starts to degrade with $13.3 \mathrm{~nm}$ scan step size. The characteristic peaks for $10 \mathrm{~nm}$ period would be expected near the edge of the Fourier modulus picture and at the $0.1 \mathrm{~nm}^{-1}$ spatial frequency on the power spectral density plot, which can be barely recognized in either location. It implies that the degradation trend starts with $13.3 \mathrm{~nm}$ scan step size and that the periodic artifact is further eliminated with $10 \mathrm{~nm}$ scanning steps. We note that the FWHM dimension of the primary mode from the reconstruction with $10 \mathrm{~nm}$ scanning step is about $16 \times 17 \mathrm{~nm}$. It appears that the scan step size has to be smaller than this FWHM size of the probe to start mitigating the periodic artifact, which is consistent with the simulation result. With $13.3 \mathrm{~nm}$ scan step size, the overlap ratio is about $70 \%$, which also agrees with the number $\sim 63 \%$ obtained in the simulation.

The periodic artifact associated with the periodicity of the scan pattern fundamentally arises from the fact that the far-field diffraction pattern measured in ptychography experiments is determined by the product of the object and the probe functions. Post-reconstruction image processing methods may be able to remove the grid artifact to some extent $[34,35]$. One approach is to mask the 

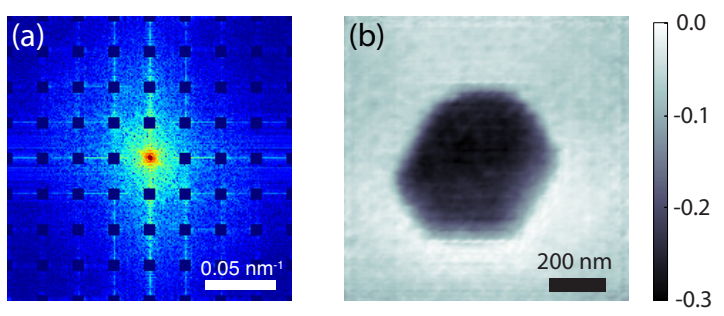

FIG. 4. Periodic artifact removal by masking satellite peaks in Fourier space. The example image is obtained with $40 \mathrm{~nm}$ scan step size. (a) The peaks associated with $40 \mathrm{~nm}$ periodicity are masked from the initial Fourier modulus as shown in Fig. 3(d). (b) The periodic artifact in the obtained realspace image is significantly reduced, compared with the initial image as shown in Fig. 2 (h).

characteristic peaks in Fourier space. Fig. 4 shows one example to apply this method to the phase image obtained with $40 \mathrm{~nm}$ scan step size. The periodic artifact is significantly removed compared with the original image shown in Fig. 2 (h). How to apply the mask nondestructively needs to be explored systematically in future studies. Completely eliminating this artifact may inevitably require to break the symmetry in the scan trajectory. Here, we present empirically that conducting the scan with sufficiently fine steps to provide over $70 \%$ overlap ratio can effectively mitigate the periodic artifact. This approach can be combined with post-reconstruction processing methods to effectively and significantly remove the periodic artifact in ptychography reconstruction. It is noticeable that it is more challenging to satisfy the stringent overlap ratio requirement with a X-ray beam focused to nanoscale. The overlap ratio has to be optimized to balance image quality and experiment throughput. Simulation also shows that the mitigation of the periodic artifact is less effective if a Gaussian-shape probe is used. It suggests a structured beam profile is preferred for ptychography reconstruction, which is consistent with experimental findings $[36,37]$.

Our demonstrated ability to obtain artifact-mitigated ptychography reconstructions using data collected in onthe-fly mode following raster scan patterns will allow seamless integration of ptychography into scanning probe microscope systems. The recovered amplitude and phase contrast images with enhanced spatial resolution provide complementary information to images obtained from other channels. Obtaining all these channels of information simultaneously and pushing the achievable resolution should significantly enhance the capability of scanning probe microscopy for a comprehensive range of applications.

\section{ACKNOWLEDGEMENT}

This research used resources of the National Synchrotron Light Source II, a U.S. Department of Energy (DOE) Office of Science User Facility operated for the DOE Office of Science by Brookhaven National Laboratory under Contract No. DE-SC0012704. H.Ö. acknowledges the support of Laboratory Directed Research and Development (LDRD-21690) program from the Brookhaven National Laboratory.

* Corresponding author; xjhuang@bnl.gov

[1] H. Mimura, S. Handa, T. Kimura, H. Yumoto, D. Yamakawa, H. Yokoyama, S. Matsuyama, K. Inagaki, K. Yamamura, Y. Sano, K. Tamasaku, Y. Nishino, M. Yabashi, T. Ishikawa, and K. Yamauchi. Breaking the $10 \mathrm{~nm}$ barrier in hard-X-ray focusing. Nature Physics, 6(2):122-125, 2010.

[2] C. Chang and A. Sakdinawat. Ultra-high aspect ratio high-resolution nanofabrication for hard x-ray diffractive optics. Nature Communications, 5:4243, 2014.

[3] I. Mohacsi, I. Vartiainen, B. Rosner, M. Guizar-Sicairos, V. Guzenko, I. McNulty, R. Winarski, M. Holt, and C. David. Interlaced zone plate optics for hard X-ray imaging in the $10 \mathrm{~nm}$ range. Scientific Reports, 7:43624, 2017.

[4] X. Huang, H. Yan, E. Nazaretski, R. Conley, N. Bouet, J. Zhou, K. Lauer, L. Li, D. Eom, D. Legnini, R. Harder, I. Robinson, and Y. Chu. $11 \mathrm{~nm}$ hard X-ray focus from a large-aperture multilayer Laue lens. Scientific Reports, 3:3562, 2013.

[5] A. Morgan, M. Prasciolu, A. Andrejczuk, J. Krzywinski, A. Meents, D. Pennicard, H. Graafsma, A. Barty, R. Bean, M. Barthelmess, D. Oberthuer, O. Yefanov, A. Aquila, H. Chapman, and S. Bajt. High numerical aperture multilayer Laue lenses. Scientific Reports, 5:9892, 2015 .

[6] M. Holler, J. Raabe, A. Diaz, M. Guizar-Sicairos, C. Quitmann, A. Menzel, and O. Bunk. An instrument for 3D x-ray nano-imaging. Review of Scientific Instruments, 83:073703, 2012.

[7] S. Chen, J. Deng, Y. Yuan, C. Flachenecker, R. Mak, B. Hornberger, Q. Jin, D. Shu, B. Lai, J. Maser, C. Roehrig, T. Paunesku, S. Gleber, D. Vine, L. Finney, J. VonOsinski, M. Bolbat, I. Spink, Z. Chen, J. Steele, D. Trapp, J. Irwin, M. Feser, E. Snyder, K. Brister, C. Jacobsen, G. Woloschak, and S. Vogt. The Bionanoprobe: hard x-ray fluorescence nanoprobe with cryogenic capabilities. Journal of Synchrotron Radiation, 21:66-75, 2014.

[8] E. Nazaretski, K. Lauer, H. Yan, N. Bouet, J. Zhou, R. Conley, X. Huang, W. Xu, M. Lu, K. Gofron, S. Kalbfleisch, U. Wagner, C. Rau, and Y. Chu. Pushing the limits: an instrument for hard X-ray imaging below $20 \mathrm{~nm}$. Journal of Synchrotron Radiation, 22:336-341, 2015.

[9] J. Rodenburg, A. Hurst, A. Cullis, B. Dobson, F. Pfeiffer, O. Bunk, C. David, K. Jefimovs, and I. Johnson. Hard-xray lensless imaging of extended objects. Physical Review 
Letters, 98:034801, 2007.

[10] A. Menzel, C. Kewish, P. Kraft, B. Henrich, K. Jefimovs, J. Vila-Comamala, C. David, M. Dierolf, P. Thibault, F. Pfeiffer, and O. Bunk. Scanning transmission X-ray microscopy with a fast framing pixel detector. Ultramicroscopy, 110:1143-1147, 2010.

[11] P. Thibault, M. Dierolf, A. Menzel, O. Bunk, C. David, and F. Pfeiffer. High-resolution scanning x-ray diffraction microscopy. Science, 321:379-382, 2008.

[12] M. Guizar-Sicairos and J. Fienup. Phase retrieval with transverse translation diversity: a nonlinear optimization approach. Optics Express, 16(10):7264-7272, 2008.

[13] A. Maiden and J. Rodenburg. An improved ptychographical phase retrieval algorithm for diffractive imaging. $U l$ tramicroscopy, 109:1256-1262, 2009.

[14] J. Deng, D. Vine, S. Chen, Y. Nashed, Q. Jin, N. Phillips, T. Peterka, R. Ross, S. Vogt, and C. Jacobsen. Simultaneous cryo X-ray ptychography and fluorescence microscopy of green algae. Proceedings of the National Academy of Sciences, 112:2314-2319, 2015.

[15] D. Shapiro, Y. Yu, T. Tyliszczak, J. Cabana, R. Celestre, W. Chao, K. Kaznatcheev, A. Kilcoyne, F. Maia, S. Marchesini, Y. Meng, T. Warwick, L. Yang, and H. Padmore. Chemical composition mapping with nanometre resolution by soft X-ray microscopy. Nature Photonics, 8:765-769, 2014.

[16] D. Vine, D. Pelliccia, C. Holzner, S. Baines, A. Berry, I. McNulty, S. Vogt, A. Peele, and K. Nugent. Simultaneous X-ray fluorescence and ptychographic microscopy of Cyclotella meneghiniana. Optics Express, 20:1828718296, 2012.

[17] J. Deng, D. Vine, S. Chen, Q. Jin, Y. Nashed, T. Peterka, S. Vogt, and C. Jacobsen. X-ray ptychographic and fluorescence microscopy of frozen-hydrated cells using continuous scanning. Scientific Reports, 7:445, 2017.

[18] H. Yan, E. Nazaretski, K. Lauer, X. Huang, U. Wagner, C. Rau, M. Yusuf, I. Robinson, S. Kalbfleisch, L. Li, N. Bouet, J. Zhou, R. Conley, and Y. Chu. Multimodality hard-x-ray imaging of a chromosome with nanoscale spatial resolution. Scientific Reports, 6:1038, 2016.

[19] J. Clark, X. Huang, R. Harder, and I. Robinson. Continuous scanning mode for ptychography,. Optics Letters, 39:6066-6069, 2014.

[20] P. Pelz, M. Guizar-Sicairos, P. Thibault, I. Johnson, M. Holler, and A. Menzel. On-the-fly scans for X-ray ptychography. Applied Physics Letters, 105:251101, 2014.

[21] X. Huang, K. Lauer, J. Clark, W. Xu, E. Nazaretski, R. Harder, I. Robinson, and Y. Chu. Fly-scan ptychography. Scientific Reports, 5:9074, 2015.

[22] J. Deng, Y. Nashed, S. Chen, N. Phillips, T. Peterka, R. Ross, S. Vogt, C. Jacobsen, and D. Vine. Continuous motion scan ptychography: characterization for increased speed in coherent x-ray imaging. Optics Express, 23:5438-5451, 2015.

[23] P. Thibault, M. Dierolf, O. Bunk, A. Menzel, and F. Pfeiffer. Probe retrieval in ptychographic coherent diffractive imaging. Ultramicroscopy, 109:338-343, 2009.

[24] M. Dierolf, P. Thibault, A. Menzel, C. Kewish, K. Jefimovs, I. Schlichting, K. Kong, O. Bunk, and F. Pfeiffer. Ptychographic coherent diffractive imaging of weakly scattering specimens. New Journal of Physics, 12:035017, 2010.

[25] A. Maiden, M. Humphry, F. Zhang, and J. Rodenburg. Superresolution imaging via ptychography. Journal of the Optical Society of America A, 28:604-612, 2011.

[26] X. Huang, H. Yan, R. Harder, Y. Hwu, I. Robinson, and Y. Chu. Optimization of overlap uniformness for ptychography. Optics Express, 22:12634-12644, 2014.

[27] O. Bunk, M. Dierolf, S. Kynde, I. Johnson, O. Marti, and F. Pfeiffer. Influence of the overlap parameter on the convergence of the ptychographical iterative engine. Ultramicroscopy, 108:481-487, 2008.

[28] T. Edo, D. Batey, A. Maiden, C. Rau, U. Wagner, Z. Pesic, T. Waigh, and J. Rodenburg. Sampling in x-ray ptychography. Physical Review A, 87:053850, 2013.

[29] J. da Silva and A. Menzel. Elementary signals in ptychography. Optics Express, 23:33812-33821, 2015.

[30] P. Thibault and A. Menzel. Reconstructing state mixtures from diffraction measurements. Nature, 494:68-71, 2013.

[31] J. Clark, X. Huang, R. Harder, and I. Robinson. Dynamic imaging using ptychography. Physical Review Letters, 112:113901, 2014.

[32] Y. Chu, H. Yan, E. Nazaretski, S. Kalbfleisch, X. Huang, K. Lauer, and N. Bouet. Hard x-ray nanoprobe facility at the National Synchrotron Light Source II. SPIE Newsroom, 31 August 2015.

[33] E. Nazaretski, H. Yan, K. Lauer, X. Huang, W. Xu, S. Kalbfleisch, H. Yan, L. Li, N. Bouet, J. Zhou, D. Shu, R. Conley, and Y. Chu. Nm-scale spatial resolution xray imaging with MLL nanofocusing optics: instrumentational requirements and challenges. AIP Conference Proceedings, 1764:040001, 2016.

[34] C. Lin, W. Lee, S. Chen, C. Tsai, J. Lee, C. Chang, and Y. Ching. A study of grid artifacts formation and elimination in computed radiographic images. Journal of Digital Imaging, 19:351-361, 2006.

[35] H. Tang, D. Tong, X. Bao, and J. Dillenseger. A new stationary gridline artifact suppression method based on the 2D discrete wavelet transform. Medical Physics, 42:17211729, 2015.

[36] M. Guizar-Sicairos, M. Holler, A. Diaz, J. VilaComamala, O. Bunk, and A. Menzel. Role of the illumination spatial-frequency spectrum for ptychography. Physical Review B, 86:100103R, 2012.

[37] A. Maiden, G. Morrison, B. Kaulich, A. Gianoncelli, and J. Rodenburg. Soft X-ray spectromicroscopy using ptychography with randomly phased illumination. Nature Communications, 4:1669, 2013. 